\section{IMMUNOPATHOGENESIS} \section{Molecular mimicry in MS}

Nature https://doi.org/10.1038/s41586-02204432-7 (2022)

Accumulating epidemiological evidence suggests a link between infection with Epstein-Barr virus (EBV) and multiple sclerosis (MS). In Nature, Lanz et al. identify pathogenic antibodies directed against the EBV nuclear antigen EBNA1 that cross-react with glial cell adhesion protein (GlialCAM) found in the central nervous system (CNS). Patients with MS have increased frequencies of B cell plasmablasts and $\mathrm{IgG}$ in cerebrospinal fluid as compared with healthy controls. B cell receptor sequencing analysis and the generation of monoclonal antibodies from these plasmablasts revealed their recognition of EBV-derived proteins and related herpesviruses. In-depth analysis of one such antibody identified its binding motif within EBNA1 as well as that of GlialCAM, whose binding interaction is vastly increased by serine phosphorylation. Whereas germline BCR sequences can recognize the EBNA1 motif, recognition of GlialCAM increases by somatic hypermutation within the CNS. Examination of other cohorts of patients with MS confirms the presence of anti-GlialCAM antibodies, and mouse models support the hypothesis that these antibodies contribute to CNS immunopathology. These findings suggest molecular mimicry between EBNA1 and host GlialCAM contributes to the development of MS.

$L A D$

https://doi.org/10.1038/s41590-022-01156-8

\section{CD4+ LYMPHOCYTES}

\section{Editing resting cells}

Nat. Methods https://doi.org/10.1038/s41592021-01328-8 (2021)

Genetic manipulation of resting $\mathrm{CD} 4^{+}$ T cells has been very technically challenging and this has limited some of the insights that can be made about the viral infection of these cells. In Nature Methods, Keppler and colleagues describe an optimized protocol that enables the gene editing of truly resting $\mathrm{CD}^{+}{ }^{+} \mathrm{T}$ cells. Human $\mathrm{CD} 4^{+} \mathrm{T}$ cells incubated at low densities on flat-bottomed plates and in medium supplemented with low-dose IL-7 plus IL-15 remain in a resting state and viable for up to 6 weeks. $\mathrm{CD}^{+}$ $T$ cells incubated in this manner can be gene edited using a CRISPR approach with near $100 \%$ efficiency. As a proof of principle, the authors knock out up to six genes simultaneously - including five that have important roles in HIV infection.

\title{
MUCOSAL IMMUNOLOGY
}

\section{Mucus needs GSDMD}

GSDMD can punch pores in macrophage cell membranes, which results in pyroptosis and the release of IL-1 and IL-18. New data show that GSDMD also has a role in maintaining the mucus layer that separates the intestinal epithelial layer from bacteria. Zhang et al. found that mice with GSDMD deleted specifically in epithelial cells (with villin-Cre) had a reduced intestinal inner mucus layer as a result of a failure of goblet cells to secrete mucus granules. Using a GSDMD-deficient human colonic goblet cell line, the researchers showed that GSDMD pores are needed for $\mathrm{Ca}^{2+}$ influx, which drives scinderin function. Scinderin, in turn, drives the remodeling of $F$-actin, which is central to mucin granule exocytosis. Intriguingly, this mechanism seems to be independent of pyroptotic cell death.

https://doi.org/10.1038/s41590-022-01155-9

This modified incubation protocol can also be used to enable knock-in of an eGFP reporter to specific loci and enable the study of HIV infection.

$Z F$

https://doi.org/10.1038/s41590-022-01154-w

\section{COVID-19}

\section{Disease trajectories}

Cell https://doi.org/10.1016/j.cell.2022.01.014 (2022)

Recovery from COVID-19 can take a long time. In Cell, Su et al. perform multi-omics profiling in a cohort of 209 patients with COVID-19 (71\% hospitalization rate) at clinical diagnosis (T1), acute disease (T2) and 2-3 months after disease onset (T3). At T3, 44\% of patients were positive for the 6 autoantibodies tested (IFN- $\alpha 2$ and nuclear proteins), with half of them positive at $\mathrm{T} 1$ (94\% at subclinical levels). T3 autoantibodies associated with inflammation biomarkers at T2 and negatively correlated with titers of SARS-CoV-2 IgG. T3 respiratory symptoms associated with IFN- $\alpha 2$ autoantibodies; neurological symptoms with SARS-CoV-2 IgG titers; fatigue with Epstein-Barr virus reactivation at $\mathrm{T} 1$; and gastrointestinal symptoms with bystander activation of CMV-specific CD8 ${ }^{+}$T cells. Single-cell RNA sequencing sorted patients into four groups: a type 1 , enriched in $\mathrm{T}_{\mathrm{H}} 1, \mathrm{M} 1$ and cytotoxic signatures in T cells, monocytes and NK cells, and memory signatures in B cells; type 2, enriched in $\mathrm{T}_{\mathrm{H}} 2, \mathrm{M} 2$ and plasma $\mathrm{B}$ cell signatures; intermediate (between types 1 and 2); and (T and NK cell) naive, all four with high monocyte polyfunctionality. The T3 type was anticipated by the transcriptomic signature at $\mathrm{T} 1$, which indicates that patients may be predisposed towards their post-acute status early in the infection.

https://doi.org/10.1038/s41590-022-01157-7

Nicholas J. Bernard, Laurie A. Dempsey, Zoltan Fehervari and Ioana Visan 